\title{
ON LOCAL TIME FOR MARKOV CHAINS
}

\author{
BY DAVID WILLIAMS ${ }^{1}$
}

Communicated by M. Loève, November 28, 1966

The object of this paper is to present one of a class of formulae which express the time spent in an instantaneous state of a Markov chain in terms of the time spent in "neighbouring" states. Though these formulae do lead to new analytic results-some elementary consequences are given in [3] - their main use is in the analysis of sample function behaviour in the neighbourhood of an instantaneous state. Time is always shared out "properly" among states in such a neighbourhood. Though the particular theorem stated below refers to a real state $b$, it has a valid extension to the case when $b$ is an instantaneous fictitious state in the sense of Neveu [2]. A detailed account of these topics will appear elsewhere.

The terminology and notation used here are exactly as in Chung [1]; see particularly the appendix for the definition of the functions $g$ and $G$.

Suppose that $\{x(t): t \geqq 0\}$ is a Borel measurable, well-separable M.C. with minimal state-space $I$. For any state $k$ in $I$, define

$$
\beta_{k}(t, \omega)=\mu\{u: 0 \leqq u \leqq t, x(u, \omega)=k\},
$$

$\mu$ denoting Lebesgue measure.

Theorem. Suppose that $b$ is an instantaneous state of $\{x(t)\}$. Suppose also that $\left\{H_{n}\right\}$ is a sequence of subsets of $I-\{b\}$ and that $\left\{s_{n}\right\}$ is a sequence of positive real numbers such that, as $n \rightarrow \infty$,

$$
s_{n} \downarrow 0, \quad \sum_{j \in H_{n}} g_{b j}\left(s_{n}\right) \rightarrow \infty .
$$

Then, for every $t$,

$$
\lim _{n \rightarrow \infty} \frac{\sum_{j \in H_{n}} g_{b j}\left(s_{n}\right) \beta_{j}(t, \omega) / G_{b j}(\infty)}{\sum_{j \in H_{n}} g_{b j}\left(s_{n}\right)}=\beta_{b}(t, \omega)
$$

in probability.

Note. Sequences $\left\{H_{n}\right\}$ and $\left\{s_{n}\right\}$ with the stated properties must

1 This work was done at the University of Durham, England. 
exist. For example, each $H_{n}$ may be taken to be $I-\{b\}$ and $\left\{s_{n}\right\}$ any sequence tending to zero.

The theorem may be proved by a Tchebycheff argument based on the following calculations.

For $t<\beta_{b}(\infty, \omega)$, define $\rho(t, \omega)$ by the equation

$$
\rho(t, \omega)=\inf \left\{s: \beta_{b}(s, \omega)>t\right\} .
$$

Then, for $j \neq b$ and $k \neq b$,

$$
E\left\{\beta_{j}(\rho(t)) \mid \beta_{b}(\infty)>t ; x(0)=b\right\}=G_{b j}(\infty) F_{j b}(\infty) t
$$

and

$$
\begin{gathered}
\operatorname{Cov}\left\{\beta_{j}(\rho(t)), \beta_{k}(\rho(t)) \mid \beta_{b}(\infty)>t ; x(0)=b\right\} \\
{\left[G_{b j}(\infty)_{b} P_{j k}(\infty) F_{k b}(\infty)+G_{b k}(\infty)_{b} P_{k j}(\infty) F_{j b}(\infty)\right] t .}
\end{gathered}
$$

The motivation for the theorem was Lévy's formula:

$$
\lim _{b \downarrow a}(\mu\{s: 0 \leqq s \leqq t, a \leqq x(s) \leqq b\}) / 2(b-a)=T(t, a)
$$

for Brownian local time.

\section{REFERENCES}

1. K. L. Chung, Markov chains with stationary transition probabilities, SpringerVerlag, Berlin, 1960.

2. J. Neveu, Sur les états d'entrée et les étates fictifs d'un processus de Markov, Ann. Inst. Henri Poincare 17 (1962), 323-337.

3. D. Williams, $A$ note on the Q-matrices of Markov chains, $Z$. Wahrscheinlichkeitstheorie 7 (1967), 116-121.

Clare College, Cambridge. 